\title{
Correlation between the use of antibiotics and development of a resistant bacterial infection in patients in the ICU
}

\author{
Yingxia $\mathrm{Li}^{1}$, Xiyan $\mathrm{Xia}^{2}$, Xiaohui $\mathrm{Li}^{3}$, Ke Xiao ${ }^{3}$, Xuewei Zhuang ${ }^{3, *}$ \\ ' General Surgery, Qilu Hospital Affiliated with Shandong University, Ji'nan, China; \\ ${ }^{2}$ Department of Immunology, Jinan Vocational College of Nursing, Ji'nan, China; \\ ${ }^{3}$ Clinical Laboratory, Qilu Hospital Affiliated with Shandong University, Ji'nan, China.
}

\begin{abstract}
Summary The current study analyzed the correlation between the use of antibiotics and development of a resistant bacterial infection in $\mathbf{4 5 4}$ patients in intensive care units (ICUs), and this study also examined factors related to development of an infection in order to facilitate more rational use of antibiotics and to reduce the incidence of resistant bacterial infections. Potential subjects were patients who were admitted to the ICU in 2016, and 454 such patients were selected using cluster sampling. Patient information was documented using an original questionnaire, Patients in the ICU with a Resistant Bacterial Infection. The correlation between use of an antibiotic and development of a resistant bacterial infection was examined. The rate of infection significantly increased over time and with receipt of various antibiotics. The development of a resistant bacterial infection was found to correlate with the use of antibiotics. Antibiotics should be used more carefully to reduce the incidence of resistant bacterial infections
\end{abstract}

Keywords: Patients in the ICU, antibiotics, resistant bacterial infection

Antibiotic resistance due to the severe overuse of antibiotics has become a worldwide problem. The global increase in infections with resistant bacteria is a cause for concern because these highly resistant bacteria are associated with higher patient morbidity and because these bacteria are susceptible to few or no antimicrobials (1-3). The Chinese Ministry of Health's Department of Medical Administration has conducted several trials to reduce the overuse of antibiotics, but more than $20 \%$ inpatients still develop a resistant bacterial infection. This has a serious impact on infection control efforts due to the lack of effective antibiotics, and especially in intensive care units (ICUs) (4). The current study examined the correlation between the use of antibiotics and development of a resistant bacterial infection and related factors in order to promote more rational use of antibiotics to reduce the development of resistant bacterial infections.

Potential subjects were patients age 18 or over who

\footnotetext{
*Address correspondence to:

Dr. Xuewei Zhuang, Clinical Laboratory Medicine, Qilu Hospital Affiliated with Shandong University, 107 Wenhua West Road, Ji'nan, Shandong 250012, China.

E-mail: zhuangxuewei@sdu.edu.cn
}

were admitted to the ICU at this Hospital no more than $48 \mathrm{~h}$ prior in 2016. Subjects were 454 such patients who were selected using cluster sampling. All subjects provided written informed consent prior to participation in this study, and this study was approved by the ethics committee at this hospital. Basic patient information was documented using an original questionnaire. Infection surveillance was achieved using a nosocomial infection surveillance system (NISS) and a hospital information system (HIS) as well as a point-of-care investigation. Data were analyzed using the statistical software SPSS 19.0. Basic information is expressed as the mean \pm standard deviation, the rate, the percentage, etc. Continuous variables were compared using the Student $t$ test and categorical variables were compared using the chi-square test. A difference of $p<0.05$ was considered statistically significant.

Of the 454 patients, 427 received an antibiotic during hospitalization. One hundred and thirty of the 427 patients $(30.44 \%)$ who received an antibiotic developed a resistant bacterial infection while 1 of the 27 patients $(3.70 \%)$ who did not receive antibiotics developed a resistant bacterial infection. The rate of infection differed significantly between the two groups $(p=0.003)($ Table 1$)$. 
Table 1. Infection with a resistant bacterium and use of an antibiotic

\begin{tabular}{|c|c|c|c|c|c|c|}
\hline \multirow{2}{*}{ Antibiotics used } & \multicolumn{2}{|c|}{ Developed a resistant bacterial infection $(n)$} & \multirow{2}{*}{ Total } & \multirow{2}{*}{ Rate of infection $(\%)$} & \multirow{2}{*}{$\chi^{2}$} & \multirow{2}{*}{$p$} \\
\hline & Yes & No & & & & \\
\hline No & 1 & 26 & 27 & 3.70 & 8.846 & $0.003 *$ \\
\hline Yes & 130 & 297 & 427 & 30.44 & & \\
\hline
\end{tabular}

Table 2. Number of antibiotics and development of a resistant bacterial infection

\begin{tabular}{|c|c|c|c|c|c|c|}
\hline \multirow{2}{*}{ Number of antibiotics } & \multicolumn{2}{|c|}{ Developed a resistant bacterial infection $(n)$} & \multirow{2}{*}{ Total } & \multirow{2}{*}{ Rate of infection $(\%)$} & \multirow{2}{*}{$\chi^{2}$} & \multirow{2}{*}{$p$} \\
\hline & Yes & No & & & & \\
\hline One & 12 & 165 & 177 & 6.78 & 152.779 & 0.000 \\
\hline Two & 38 & 100 & 138 & 27.54 & & \\
\hline Three & 31 & 27 & 58 & 53.45 & & \\
\hline Four or more & 48 & 5 & 53 & 90.57 & & \\
\hline
\end{tabular}

The rate of infection with a resistant bacterium differed significantly in patients who had received one, two, three, or four or more antibiotics, indicating that the rate of infection increased significantly when patients received a combination of antibiotics (Table 2).

Of the 454 patients, 298 patients had previously received an antibiotic before admission to the ICU. One hundred and twelve of those 298 patients $(37.58 \%)$ subsequently developed a resistant bacterial infection. Only 18 of the 156 remaining patients $(11.54 \%)$ developed a resistant bacterial infection. The rate of infection in those two groups different significantly ( $p$ $<0.001)$. Patients who received an antibiotic before admission to the ICU developed a resistant bacterial infection at a rate of $37.58 \%$, which was significantly higher than the rate in patients who had not received an antibiotic $(p<0.001)$. Fifty-four of the 454 patients died, and 34 of those patients had a resistant bacterial infection. Only 97 of the 400 surviving patients had a resistant bacterial infection. The rate of infection in patients who died was $62.96 \%$, which was significantly higher than the rate in patients who survived $(p<0.001)$.

Infections are effectively regulated as a result of the large-scale use of antibiotics. However, the overuse of antibiotics has led to strains developing antibiotic resistance and even causing reinfection. Moreover, infections rage out of control and mortality increases when host bacterial homeostasis in vivo is disrupted (3). Patients with an infection have more severe subsequent complications and a higher mortality, and unwarranted use of antibiotics results in a vicious circle of resistant bacterial infections $(5,6)$. Hence, antibiotic resistance has garnered attention around the world. The current study examined the correlation between use of an antibiotic and development of a resistant bacterial infection in detail. Patients who had previously received an antibiotic developed a resistant bacterial infection at a higher rate than patients who had not received antibiotics. The rate of infection increased over time and with previous receipt of an antibiotic. Patients in the ICU who had previous received an antibiotic had a significantly higher rate of infection than patients who had not received antibiotics. The incidence of a resistant bacterial infection was correlated with the receipt of an antibiotic, and especially a broad-spectrum antibiotic. Infection with an antibiotic-resistant bacterium is one of the leading causes of mortality (7).

In summary, the current study found that development of a resistant bacterial infection correlated with the use of antibiotics. Antibiotics should be used more carefully to reduce the incidence of resistant bacterial infections. Medical personnel should adopt certain principles to reduce resistant bacterial infections as much as possible, such as rational prescribing based on a drug's properties, combating infections based on a drug sensitivity test, proper dosage over the required period, careful attention to normal bacteria, use of certain routes of medications, and avoiding blind usage and frequent alternations in medication (8).

\section{Acknowledgements}

This study was supported in part by grants from the Shandong Provincial Key Research Program (2016GSF201169), the Shandong Provincial Nature Science Foundation (2015ZRE2757), the Shandong Provincial Medicine and Health Development Plan (QW019), and the Shandong Provincial Population and Family Development Plan (200910).

\section{References}

1. Haverkate MR, Derde LP, Brun-Buisson C, Bonten MJ, Bootsma MC. Duration of colonization with antimicrobialresistant bacteria after ICU discharge. Intensive Care Med. 2014; 40:564-571.

2. Magiorakos AP, Burns K, Rodríguez Baño J, Borg M, Daikos G, Dumpis U, Lucet JC, Moro ML, Tacconelli E, Simonsen GS, Szilágyi E, Voss A, Weber JT. 
Infection prevention and control measures and tools for the prevention of entry of carbapenem-resistant Enterobacteriaceae into healthcare settings: Guidance from the European Centre for Disease Prevention and Control. Antimicrob Resist Infect Control. 2017; 6:113.

3. Martin-Loeches I, Torres A, Rinaudo M, Terraneo S, de Rosa F, Ramirez P, Diaz E, Fernández-Barat L, Li Bassi GL, Ferrer M. Resistance patterns and outcomes in intensive care unit (ICU)-acquired pneumonia. Validation of European Centre for Disease Prevention and Control (ECDC) and the Centers for Disease Control and Prevention (CDC) classification of multidrug resistant organisms. J Infect. 2015; 70:213-222.

4. Hanberger H, Walther S, Leone M, Barie PS, Rello J, Lipman J, Marshall JC, Anzueto A, Sakr Y, Pickkers P, Felleiter P, Engoren M, Vincent JL; EPIC II Group of Investigators. Increased mortality associated with methicillin-resistant Staphylococcus aureus (MRSA) infection in the intensive care unit: Results from the EPIC
II study. Int J Antimicrob Agents. 2011; 38:331-335.

5. Harris AD, Pineles L, Belton B, et al. Universal glove and gown use and acquisition of antibiotic-resistant bacteria in the ICU: A randomized trial. JAMA. 2013; 310:15711580.

6. Derde LP, Dautzenberg MJ, Bonten MJ. Chlorhexidine body washing to control antimicrobial-resistant bacteria in intensive care units: A systematic review. Intensive Care Med. 2012; 38:931-939.

7. Shafi S, Collinsworth AW, Richter KM, et al. Bundles of care for resuscitation from hemorrhagic shock and severe brain injury in trauma patients-translating knowledge into practice. J Trauma Acute Care Surg. 2016; 81:780-794.

8. Yang A, Tang WS, Si T, Tang JX. Influence of physical effects on the swarming motility of Pseudomonas aeruginosa. Biophys J. 2017; 112:1462-1471.

(Received June 12, 2018; Revised September 3, 2018; Accepted October 6, 2018) 